\title{
Posterior Reversible Encephalopathy Syndrome in End-Stage Kidney Disease: Not Strictly Posterior or Reversible
}

\author{
Mark Canney Dearbhla Kelly Michael Clarkson \\ Department of Renal Medicine, Cork University Hospital, Cork, Ireland
}

\section{Key Words}

Posterior reversible encephalopathy syndrome - End-stage

kidney disease $\cdot$ Haemodialysis - Magnetic resonance

imaging $\cdot$ Hypertension

\begin{abstract}
Posterior reversible encephalopathy syndrome (PRES) is an uncommon clinico-radiological condition that can result in severe brain injury. The pathogenesis of cerebral vasogenic edema, the hallmark of PRES, is not fully understood. Despite its name, there is substantial heterogeneity both in terms of imaging findings and outcome. Relatively little is known about PRES in kidney disease despite the clustering of risk factors including hypertension, autoimmune disease and immunosuppression. In a retrospective observational study of incident end-stage kidney disease patients in Southwest Ireland over a ten year period, we discovered five cases of PRES representing an incidence of $0.84 \%$ in this patient population. These five cases highlight the variability in clinical presentation and the potentially life-threatening nature of this condition. We provide an in-depth review of the existing literature regarding PRES in terms of its pathogenesis and heterogeneity, as well as the experience of PRES in ESKD patients. PRES appears to be rare in the ESKD population but could be under-recognized. Marked hypertension is a cardinal risk factor in this population, associated with extracellular fluid volume expansion. Neuroimaging findings can be
\end{abstract}

diverse involving both anterior and posterior circulation territories. Three of the five patients described had commenced haemodialysis within four weeks of their presentation. These patients may be particularly vulnerable to microvascular brain injury, which can be devastating. This emphasises the need for clinicians to pay meticulous attention to extracellular fluid volume control during this potentially hazardous period.

C 2015 S. Karger AG, Basel

\section{Introduction}

Posterior reversible encephalopathy syndrome (PRES) is a clinico-radiological disorder characterised by the abrupt onset of neurological symptoms associated with potentially reversible lesions on brain imaging. The differential diagnosis is broad due to the heterogeneity of symptoms which include seizure, headache, visual disturbance and altered mental status. Diagnosis is made using computerised tomography (CT) or, most commonly, magnetic resonance imaging (MRI), the latter using diffusion weighted imaging (DWI). The epidemiology of PRES is largely unknown due to the paucity of large prospective series. Although PRES can affect people of all ages, the median age at presentation is approximately 40 years based on existing registry data $[1,2]$. Females appear to be affected more than males [1].

\section{KARGER 125}

(C) 2015 S. Karger AG, Basel

0250-8095/15/0413-0177\$39.50/0

E-Mail karger@karger.com

www.karger.com/ajn
Dr. Mark Canney

9 Camac Way, Kearns Place

Old Kilmainham, Dublin 8 (Ireland)

E-Mail mark_canney@hotmail.com 
Table 1. Summary of individual patient characteristics

\begin{tabular}{|c|c|c|c|c|c|}
\hline Age, years & 15 & 64 & 43 & 20 & 56 \\
\hline Cause of ESKD & $\begin{array}{l}\text { ANCA-associated } \\
\text { vasculitis }\end{array}$ & Hypertension & Anephric & Lupus nephritis & $\begin{array}{l}\text { Diabetic } \\
\text { nephropathy }\end{array}$ \\
\hline $\begin{array}{l}\text { Recent initiation of } \mathrm{HD} \\
(<4 \text { weeks })\end{array}$ & Yes & No & Yes & Yes & No \\
\hline Recent immunosuppression & Yes & No & No & Yes & No \\
\hline Clinical presentation & Seizures & Ataxia & Headache, seizures & Headache, seizures & $\begin{array}{l}\text { Altered mental } \\
\text { state }\end{array}$ \\
\hline $\mathrm{BP}$ (MAP), mm Hg & $150 / 110(123)$ & $198 / 140(159)$ & $180 / 111(134)$ & $162 / 114(130)$ & $202 / 91(128)$ \\
\hline Cerebral involvement & $\begin{array}{l}\text { Bilateral frontal } \\
\text { and parietal lobes }\end{array}$ & $\begin{array}{l}\text { Left parietal and } \\
\text { bilateral } \\
\text { occipital lobes }\end{array}$ & $\begin{array}{l}\text { Left frontal and } \\
\text { parietal } \\
\text { lobes (haemorrhagic) }\end{array}$ & $\begin{array}{l}\text { Right occipital, left } \\
\text { parieto-occipital } \\
\text { lobes }\end{array}$ & Right hemisphere \\
\hline
\end{tabular}

ANCA = Anti-neutrophil cytoplasmic antibody; BP = blood pressure; ESKD = end-stage kidney disease; HD = haemodialysis; $\mathrm{MAP}=$ mean arterial pressure.

The nomenclature of this condition has been the subject of much debate ever since its original description in 1996 [3]. Hinchey et al. observed a consistent pattern in both neurological symptoms and brain imaging findings in 15 patients and coined the term 'Reversible Posterior Leukoencephalopathy Syndrome'. The nomenclature changed over subsequent years to 'PRES' in an effort to popularise the diagnosis and reflect its association with elevated blood pressure [4]. However, the appropriateness of the acronym PRES has been questioned, specifically with regard to the reversibility of the lesions and the anatomical restriction to the posterior compartment of the brain [5].

There have been scattered reports of PRES in kidney disease but it has never been researched in a systematic fashion. In Hinchey's original paper, over half of the cases had an abnormal serum creatinine, one third had a serum creatinine over $3 \mathrm{mg} / \mathrm{dl}(265 \mu \mathrm{mol} / \mathrm{l})$ and hypertension was present in $80 \%$ of cases. PRES has been observed in the context of many multi-system diseases that are commonly associated with kidney disease such as preeclampsia, vasculitis and systemic lupus erythematosus. PRES has also been encountered following solid organ transplantation and with the use of calcineurin inhibitors. Despite these consistent associations with kidney disease risk factors, the literature regarding PRES in individuals with established kidney disease is relatively sparse. We describe our experience of PRES in five patients with end- stage kidney disease (ESKD) along with a critical review of the existing literature. Details of the cases are summarised in table 1.

\section{Cases}

Case 1

A 15-year-old female presented with rapidly progressive glomerulonephritis secondary to granulomatous polyangiitis. Her serum creatinine at presentation was $1,191 \mu \mathrm{mol} / \mathrm{l}$ and she was commenced on haemodialysis. She received induction immunosuppression in the form of pulsed intravenous methylprednisolone in combination with plasma exchange and mycophenolate mofetil. Her symptoms improved and she was discharged home on maintenance haemodialysis three times per week for four hours. She continued on mycophenolate mofetil and oral prednisolone. Twelve days later she was readmitted with generalised tonic-clonic seizures. She was hypertensive on admission with a blood pressure (BP) of 150/110 $\mathrm{mm} \mathrm{Hg}$ (mean arterial pressure (MAP) $123 \mathrm{~mm} \mathrm{Hg}$ ). Her blood pressure was initially managed using intravenous labetalol. She was admitted to the intensive care unit and commenced on continuous veno-venous hemofiltration (CVVH). Diffusion-weighted MRI showed multiple subcortical high signal areas with restricted diffusion in both the right and left frontal and parietal lobes (fig. 1). Electro-encephalogram (EEG) demonstrated non-specific slow wave changes. Cerebrospinal fluid analysis was unremarkable. Following extracellular fluid volume control using continuous renal replacement therapy her BP gradually improved and she had no further seizures. Repeat MR imaging 3 weeks later demonstrated complete resolution of the aforementioned lesions. 


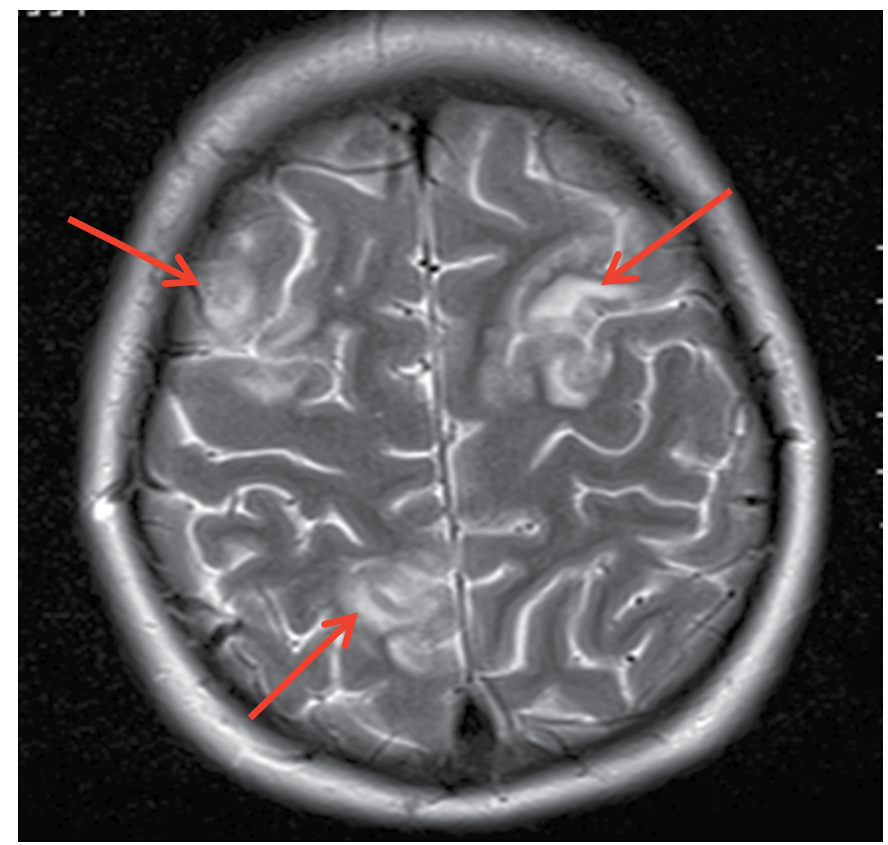

Fig. 1. T2-weighted MRI showing multiple subcortical high-signal areas in both frontal lobes and along the inter-hemispheric fissure (red arrows).

\section{Case 2}

A 64-year-old male with a background of stage 5 chronic kidney disease (CKD) and longstanding hypertension was admitted with ataxia and speech disturbance. He had stopped taking his anti-hypertensive medications in the previous 4 months. His BP on admission was 198/140 mm Hg (MAP 159 mm Hg). On examination he was found to be dysarthric and had reduced lower limb power and poor co-ordination. Serum creatinine was $940 \mu \mathrm{mol} / \mathrm{l}$ on admission and he was commenced on haemodialysis. MRI brain revealed extensive edema involving thalami, midbrain and upper pons. Scattered areas of restricted diffusion were seen in both occipital lobes and in the left parietal-occipital area. His BP was managed using a combination of anti-hypertensive medications and frequent ultrafiltration. He was discharged with full resolution of his symptoms 3 weeks later.

\section{Case 3}

A 43-year-old male presented with severe headache, vomiting, expressive dysphasia and seizures. He had a background of recurrent transitional cell carcinoma ultimately requiring bilateral nephrectomy. In the four weeks prior to this period he was established on maintenance haemodialysis. His BP post-operatively was initially low but started to increase over subsequent weeks. This was being managed by progressive reduction in dry weight with alternate day four-hour haemodialysis. His BP on admission was $180 / 111 \mathrm{~mm} \mathrm{Hg}$ (MAP $134 \mathrm{~mm} \mathrm{Hg}$ ). He was commenced on $\mathrm{CVVH}$ and parenteral antihypertensive therapy (intravenous labetalol infusion). An MRI brain revealed extensive bilateral vasogenic edema with secondary haemorrhage in the left frontal and parietal lobes with consequent subfalcine herniation. Findings were in accordance with the haemorrhagic variant of PRES. An
EEG showed an excess of diffuse slow wave activity, consistent with a moderate diffuse encephalopathy. Despite intensive ultrafiltration, anti-hypertensive and anti-seizure medications, his condition deteriorated. He died $24 \mathrm{~h}$ later from brainstem compression.

\section{Case 4}

A 20-year-old male presented to the ER with multiple generalised tonic-clonic seizures. He also complained of headache and blurred vision. He had a background of ESKD secondary to lupus nephritis. He was started on haemodialysis three weeks prior to this period and was attending the dialysis unit for four hours three days per week. The primary indication for dialysis was extracellular fluid volume expansion and poorly controlled hypertension. This was in the context of a severe systemic flare of his lupus necessitating high-dose steroids (prednisolone $40 \mathrm{mg}$ daily), nine sessions of plasma exchange and four doses of rituximab. On arrival his BP was 162/114 (MAP $130 \mathrm{~mm} \mathrm{Hg}$ ). He was poorly responsive with decreased muscle tone throughout and up-going plantar responses. A non-contrast CT brain showed foci of low attenuation involving the subcortical white matter in the right occipital lobe and left parieto-occipital region. Subsequent MRI revealed highsignal areas in the frontal lobe white matter as well as the grey and white matter of the occipital lobes bilaterally. His BP was controlled with a combination of anti-hypertensive medications and frequent ultrafiltration. Repeat MRI two weeks later demonstrated complete resolution of these abnormal areas.

\section{Case 5}

A 56-year-old lady presented with acute onset confusion, vomiting and myoclonus. She had a background of ESKD, hypertension, coronary artery disease and longstanding type 1 diabetes mellitus. She had been receiving intermittent haemodialysis for three-and-ahalf hours 3 days per week for 2 years. Her blood pressure was being managed with a combination of ramipril, amlodipine and bisoprolol. Shortly into her admission to hospital she had further deterioration in her mental state with fluctuating levels of alertness. This coincided with worsening hypertension (peak BP 202/91 mm Hg, MAP $128 \mathrm{~mm} \mathrm{Hg}$ ). EEG demonstrated a diffuse slow wave pattern in keeping with metabolic encephalopathy. On day 12 of her admission, she experienced multiple seizures and was transferred to the intensive care unit where she was intubated, sedated and commenced on CVVH. MRI brain revealed extensive edema with diffusion abnormalities primarily involving the right hemisphere, portions of which showed restricted diffusion suggesting foci of superimposed infarction. Her blood pressure remained labile and she continued to have seizure activity. She subsequently developed multiple organ dysfunction syndrome and died.

\section{Discussion}

\section{Pathophysiology of PRES}

Before exploring the pathogenesis of PRES, we will first revisit some concepts of cerebral blood flow. Autoregulation refers to the ability of an organ to alter its vascular resistance and maintain normal flow despite changes in arterial pressure [6]. This is particularly important in the brain, an organ that receives approximately $15 \%$ of 
Table 2. Key conceptual differences between the two proposed pathophysiological mechanisms of PRES and how they might relate to heterogeneous clinical manifestations

Hyper-perfusion hypothesis

\begin{tabular}{ll}
\hline Mechanism of injury & Systemic blood pressure exceeds upper \\
& autoregulatory threshold for cerebral blood flow; \\
& high pressure is transmitted to capillaries causing \\
endothelial injury and consequent edema
\end{tabular}

Role of high arterial pressure Primary driver of injury

Example clinical scenarios

Extremely high blood pressure e.g. volume expansion in a patient receiving dialysis

Location of cerebral injury
Often posterior brain (less sympathetic innervation), can be more diffuse
Hypo-perfusion hypothesis

Endothelial dysfunction causes intense

vasoconstriction and downstream hypo-perfusion. Capillary permeability is increased due to hypoxia resulting in edema

May be a secondary phenomenon in response to low cerebral blood flow

Systemic illness or treatment e.g. autoimmune disease, vasculitis, transplantation, calcineurin inhibitors

Can involve any part of the brain, often seen in watershed areas cardiac output. Cerebral blood flow must be maintained constant despite fluctuations in systemic arterial pressure. In the setting of low blood pressure cerebral arterioles will dilate to increase flow to the capillaries. In contrast, high blood pressure will result in arteriolar vasoconstriction to limit flow downstream. Such is the sophistication of this system, the human brain can maintain normal perfusion within a range of mean arterial pressure between approximately 60 and $140 \mathrm{~mm} \mathrm{Hg}$ [7]. Beyond these thresholds of autoregulation brain injury will occur due to hypoperfusion or hyperperfusion, respectively.

The hallmark of PRES is vasogenic edema. The advent of DWI has facilitated the differentiation of vasogenic edema from cytotoxic edema $[8,9]$, the latter an irreversible phenomenon due to tissue infarction [10]. Controversy exists, however, as to the pathogenesis of vasogenic edema in PRES and the role of hypertension. Two theories, recently reviewed by Bartynski [11], have been suggested. Table 2 provides a summary of the conceptual differences between the two hypotheses. In the most popular theory, severe systemic hypertension overwhelms the auto-regulatory capacity of the cerebral vasculature (principally arterioles) leading to hyper-perfusion, arteriolar dilatation, injury to the capillary bed and vasogenic edema. Sympathetic stimulation can raise the upper threshold of autoregulation; therefore, the predilection of PRES for the posterior brain, where there is a relative lack of sympathetic innervation, adds weight to this hypothesis. Although logical, there are a number of problems with this theory. First, blood pressure is not always elevated in PRES $[2,3,12]$, and this is particularly true in cases associated with immunosuppression [2] and organ transplantation [13, 14]. In addition, the degree of vasogenic edema does not always correlate with the severity of hypertension [13].

In the second theory, the principal problem is cerebral vasoconstriction that results in downstream hypo-perfusion, ischaemia and vasogenic edema due to capillary leak. Again the strongest argument in favour of this hypothesis is the recognition that blood pressure is either normal or minimally elevated (i.e., below the upper autoregulatory threshold) in a significant proportion of PRES cases. Hypertension therefore cannot be the sole driver of injury in all cases. Indeed PRES is often encountered in the context of a systemic process such as autoimmune disease [15], organ transplantation [13, 14] and pre-eclampsia [16]. These conditions share many pathophysiological features including upregulation of the acquired immune response and endothelial cell activation and/or injury. Endothelial cell dysfunction can lead to altered vascular tone, vasospasm, sustained hypoperfusion and ischaemia. Cerebral vessels respond to hypoxia by secreting vascular endothelial growth factor (VEGF) and increasing their permeability, thereby resulting in edema [11]. The concept of hypoperfusion is further supported by the location of injury encountered in PRES, which is consistent with the so-called watershed areas of the brain [1]. These observations support the notion that a systemic process causing endothelial dysfunction results in a cascade of events culminating in cerebral vasoconstriction, hypoperfusion and ischaemia.

Although there is some evidence to support either theory, retrospective study design, varying terminology and different imaging modalities all make it difficult to choose one theory over another. They may not be distinct from 
one another. For instance, in our cohort, although hypertension was a common feature among all five patients, the peak MAP was below the recognised upper limit of autoregulation in four cases. Other authors have proposed an upper MAP threshold of $116 \mathrm{~mm} \mathrm{Hg}$ to define clinically severe hypertension in the context of PRES $[2,12]$. Using this cut-off all five of our cases would have been classified as having severe hypertension. Two of the patients (cases 1 and 4) had a co-existent systemic inflammatory disorder with recent exposure to potent immunosuppressive agents. It is plausible that another process could reduce the upper autoregulatory threshold by altering vascular dynamics, thus increasing the potential for hypertensionmediated injury.

\section{Heterogeneity of PRES}

A key observation from the cases described earlier is the heterogeneity both in terms of lesion distribution and clinical outcome. Despite the original definition of this disorder as a posterior phenomenon, potentially all areas of the brain can be affected. The classical pattern of vasogenic edema in the parietal or occipital region is still seen in a majority of cases. Frontal lobe involvement, however, has been reported to be as high as $68 \%$ [1]. In the Berlin PRES study, involvement of frontal and temporal lobes occurred in one half of cases [2]. Lesions involving the infra-tentorial region, particularly the cerebellum, were encountered in more than half of cases.

One of our patients had a haemorrhagic variant of PRES. In large case series intracranial haemorrhage has been encountered in 15 to $32 \%$ of cases $[2,12]$. In a study of haemorrhagic PRES, seizure and confusion were the most common presenting features; however, hypertension was surprisingly not a significant factor in the development of haemorrhage [12]. Two of the five patients described died during their admission emphasizing the serious and potentially life-threatening nature of this condition. The Berlin PRES study demonstrated that, of those who had repeat imaging performed, $43 \%$ had incomplete resolution of edema on repeat MRI [2]. A significant factor associated with incomplete resolution was higher MAP at presentation. The presence of haemorrhage also portends a worse outcome [12].

\section{PRES in ESKD}

Given the fact that hypertension is a significant factor in the development of PRES, and the consistent associations of PRES with autoimmune diseases and immunosuppressive drugs, one would anticipate a high incidence of PRES in individuals with ESKD. Based on current lit- erature, this does not appear to be the case. The cases described earlier were found by interrogating the incident ESKD database of South West Ireland. Of a total of 592 cases of incident ESKD over a ten-year period, 5 cases of PRES were discovered representing an incidence of $0.84 \%$ in this population. In the larger published series of PRES, kidney disease does not feature prominently outside the realm of solid organ transplantation. PRES has been observed in a number of individuals receiving peritoneal dialysis. Most of these cases had significant hypertension at presentation and responded to $\mathrm{BP}$ control with or without ultrafiltration [17-20].

In three of the five patients described here, the onset of PRES was within weeks of commencing haemodialysis. This suggests a vulnerability period during which time patients were becoming accustomed to fluctuations in MAP by alterations to their 'dry weight.' A key learning point from these cases is the need for meticulous extracellular fluid volume and blood pressure control at the time of commencement of dialysis. Anuric patients in particular need very close monitoring and avoidance of long inter-dialytic gaps. In a recent review of three cases of PRES in haemodialysis patients, severe hypertension was the key clinical finding and responded well to strict volume control without the need for anti-hypertensive agents. Two of these patients developed PRES within three months of starting haemodialysis [21].

It is possible that pre-existing brain injury could predispose to PRES in dialysis patients. Clinically silent white matter lesions are common in individuals with kidney disease and probably reflect associated vascular disease [22]. In an interesting study of patients undergoing their first haemodialysis, MR brain imaging was performed within 30 minutes before and after the dialysis treatment [23]. Baseline imaging demonstrated a diffuse increase in apparent diffusion coefficient (ADC) in ESKD patients compared to controls indicative of interstitial edema. In the post-dialysis images, the ADC further increased particularly in the frontal lobes, suggestive of worsening interstitial edema. None of the patients who underwent haemodialysis experienced significant neurological symptoms. Brain imaging abnormalities without accompanying symptoms after dialysis has been reported elsewhere [24]. It is likely, therefore, that our dialysis patients do experience neurological sequelae of their treatment, but only a proportion comes to clinical attention. In our cases the onset of neurological symptoms was outside the dialysis or immediate post-dialysis period, and severe hypertension was the predominant feature. This, along with the particular imaging findings, favoured PRES as the diagnosis. 


\section{Transplantation}

The incidence of PRES post-transplantation varies depending on the type of organ transplanted. A retrospective single-centre study of 4222 solid organ transplants showed an overall incidence of PRES of $0.49 \%$ [13]. The incidence of PRES in kidney transplant recipients (KTRs) was $0.35 \%$. Some interesting observations emerged from this study. KTRs with PRES had much higher MAP at presentation than liver transplant recipients (LTRs) but, surprisingly, lower grades of vasogenic edema. LTRs developed PRES much earlier in the posttransplant period than KTRs, often in the setting of severe infection. This is not unexpected given the comparatively poor health status of individuals with liver disease who require emergent transplantation. In both KTRs and LTRs, PRES was diagnosed at the time of co-existent infection or organ rejection. In the context of a complex transplant recipient with infection and/or rejection, it is difficult to attribute a single aetiology to the development of PRES.

\section{Conclusion}

PRES is an uncommon entity among patients with ESKD. However, the varied clinical presentation and the increasing recognition of heterogeneous imaging findings make it difficult to be accurate about its true incidence. The reversibility of PRES has to be questioned given our experience and that of other centres. In our experience hypertension was a significant driver of brain injury, often in the setting of recent commencement of haemodialysis. This highlights the need for meticulous extracellular fluid volume control during this potentially vulnerable period.

\section{References}

1 Bartynski WS, Boardman JF: Distinct imaging patterns and lesion distribution in posterior reversible encephalopathy syndrome. AJNR Am J Neuroradiol 2007;28:1320-1327.

-2 Liman TG, Bohner G, Heuschmann PU, Endres M, Siebert E: The clinical and radiological spectrum of posterior reversible encephalopathy syndrome: the retrospective Berlin PRES study. J Neurol 2012;259:155-164.

$\checkmark 3$ Hinchey J, Chaves C, Appignani B, Breen J, Pao L, Wang A, et al: A reversible posterior leukoencephalopathy syndrome. N Engl J Med 1996;334:494-500.

4 Maizlin ZV, Ghandehari H, Maizels L, Shewchuk JR, Kirby JM, Vora P, et al: Linguistic history of posterior reversible encephalopathy syndrome: mirror of developing knowledge. J Neuroimaging 2011;21:1-4.

5 Stott VL, Hurrell MA, Anderson TJ: Reversible posterior leukoencephalopathy syndrome: a misnomer reviewed. Intern Med J 2005;35:83-90

6 Guyton AC, Hall JE: Overview of the circulation; biophysics of pressure, flow and resistance; in Textbook of Medical Physiology, ed 12. Philadelphia, Elsevier Saunders, 2011, pp 157-166.

7 Guyton AC, Hall JE: Cerebral blood flow, cerebrospinal fluid, and brain metabolism; in Textbook of Medical Physiology, ed 12. Philadelphia, Elsevier Saunders, 2011, pp 743750.

8 Mukherjee P, McKinstry RC: Reversible posterior leukoencephalopathy syndrome: evaluation with diffusion-tensor MR imaging. Radiology 2001;219:756-765.

-9 Ay H, Buonanno FS, Schaefer PW, Le DA, Wang B, Gonzalez RG, et al: Posterior leukoencephalopathy without severe hypertension: utility of diffusion-weighted MRI. Neurology 1998;51:1369-1376.

10 Covarrubias DJ, Luetmer PH, Campeau NG: Posterior reversible encephalopathy syndrome: prognostic utility of quantitative diffusion-weighted MR images. AJNR Am J Neuroradiol 2002;23:1038-1048.

11 Bartynski WS: Posterior reversible encephalopathy syndrome, part 2: controversies surrounding pathophysiology of vasogenic edema. AJNR Am J Neuroradiol 2008;29:10431049.

12 Hefzy HM, Bartynski WS, Boardman JF, Lacomis D: Hemorrhage in posterior reversible encephalopathy syndrome: imaging and clinical features. AJNR Am J Neuroradiol 2009, 30:1371-1379.

13 Bartynski WS, Tan HP, Boardman JF, Shapiro R, Marsh JW: Posterior reversible encephalopathy syndrome after solid organ transplantation. AJNR Am J Neuroradiol 2008;29:924930.

14 Cruz RJ Jr, DiMartini A, Akhavanheidari M, Iacovoni N, Boardman JF, Donaldson J, et al: Posterior reversible encephalopathy syndrome in liver transplant patients: clinical presentation, risk factors and initial management. Am J Transplant 2012;12:2228-2236.

15 Shaharir SS, Remli R, Marwan AA, Said MS, Kong NC: Posterior reversible encephalopathy syndrome in systemic lupus erythematosus: pooled analysis of the literature reviews and report of six new cases. Lupus 2013;22:492-496.

-16 Liman TG, Bohner G, Heuschmann PU, Scheel $\mathrm{M}$, Endres M, Siebert E: Clinical and radiological differences in posterior reversible encephalopathy syndrome between patients with preeclampsia-eclampsia and other predisposing diseases. Eur J Neurol 2012;19:935-943.
17 Graham BR, Pylypchuk GB: Posterior reversible encephalopathy syndrome in an adult patient undergoing peritoneal dialysis: a case report and literature review. BMC Nephrol 2014; $15: 10$.

18 Ogawa A, Sugiyama H, Nakayama K, Morinaga $\mathrm{H}$, Akagi $\mathrm{S}$, Makino $\mathrm{H}$ : Reversible posterior leukoencephalopathy syndrome in a young adult patient receiving peritoneal dialysis. Perit Dial Int 2012;32:587-589.

19 Kitamura M, Furusu A, Hirose M, Nishino T, Obata Y, Uramatsu T, et al: A case of reversible posterior leukoencephalopathy syndrome in a patient on peritoneal dialysis. Clin Exp Nephrol 2010;14:633-636.

20 Girisgen I, Tosun A, Sonmez F, Ozsunar Y: Recurrent and atypical posterior reversible encephalopathy syndrome in a child with peritoneal dialysis. Turk J Pediatr 2010;52:416-419.

21 Gungor O, Kircelli F, Kitis O, Asci G, Toz H, Ok E: Can strict volume control be the key for treatment and prevention of posterior reversible encephalopathy syndrome in hemodialysis patients? Hemodial Int 2013;17:107-110.

22 Martinez-Vea A, Salvado E, Bardaji A, Gutierrez C, Ramos A, Garcia C, et al: Silent cerebral white matter lesions and their relationship with vascular risk factors in middle-aged predialysis patients with CKD. Am J Kidney Dis 2006;47:241-250.

23 Chen CL, Lai PH, Chou KJ, Lee PT, Chung HM, Fang HC: A preliminary report of brain edema in patients with uremia at first hemodialysis: evaluation by diffusion-weighted MR imaging. AJNR Am J Neuroradiol 2007;28: 68-71.

24 Walters RJ, Fox NC, Crum WR, Taube D, Thomas DJ: Haemodialysis and cerebral oedema. Nephron 2001;87:143-147. 